
\title{
$\begin{array}{ll}\text { Research Square } & \begin{array}{l}\text { Preprints are preliminary reports that have not undergone peer review. } \\ \text { They should not be considered conclusive, used to inform clinical practice, } \\ \text { or referenced by the media as validated information. }\end{array}\end{array}$
}

\section{Clinicopathological and Molecular Characteristics of Atypical Pulmonary Sclerosing Pneumocytoma With Multiple Metastases}

\section{Qiushi Wang}

Third Military Medical University Daping Hospital and Research Institute of Surgery

\section{Xiao Yang}

Third Military Medical University Daping Hospital and Research Institute of Surgery

Minrui Jiang

Third Military Medical University Daping Hospital and Research Institute of Surgery

\section{Mengxia Li}

Third Military Medical University Daping Hospital and Research Institute of Surgery

\section{Lei Zhang}

Genecast Precision Medision Technology Institute

\section{Yong $\mathrm{He}$}

Third Military Medical University Daping Hospital and Research Institute of Surgery

\section{Lianhua Zhao}

Third Military Medical University Daping Hospital and Research Institute of Surgery

Qiang Ma

Third Military Medical University Daping Hospital and Research Institute of Surgery Yan Chen

Third Military Medical University Daping Hospital and Research Institute of Surgery Chengyi Mao

Third Military Medical University Daping Hospital and Research Institute of Surgery

Ping Fu

Third Military Medical University Daping Hospital and Research Institute of Surgery

Ying Yang

Genecast Precision Medicine Technology Institute

Hualiang Xiao ( $\nabla$ dpbl_xhl@126.com)

Third Military Medical University Daping Hospital and Research Institute of Surgery

\section{Research}

Keywords: Pulmonary sclerosing pneumocytoma, metastasis, AKT1, TP53, copy number instability

Posted Date: July 23rd, 2020

DOI: https://doi.org/10.21203/rs.3.rs-45881/v1

License: (a) (7) This work is licensed under a Creative Commons Attribution 4.0 International License. Read Full License 


\section{Abstract}

Background: Few patients with pulmonary sclerosing pneumocytoma (PSP) may suffer from recurrence and oligometastasis as it is a benign tumor or has a low malignancy potential. Herein, an in-depth study, based on an extremely rare PSP case with atypical features, was carried out to elaborate the potential mechanism underlying the rapid malignant progression.

Methods: The clinicopathological data of this atypical PSP (AP) case were obtained. Formalin-fixed and paraffin-embedded tissues from all the lesions of AP and five classic PSP cases (as control) were used for whole-exome sequencing (WES) and immunohistochemistry.

Results: A 23-year-old male showed a 6.5-cm pulmonary nodule in the right middle lobe (RML) and enlarged mediastinal lymph nodes (LNs). He underwent thoracoscopic RML lobectomy, systematic LNs dissection, and mediastinal lymphadenectomy. The metastases to the cervical LNs and liver were detected in a short period and then resected. Postoperative pathological examination confirmed the diagnosis of PSP in all the lesions, based on the typical histological characteristics and immunophenotypes. Furthermore, WES identified both AKT1 E17K somatic mutation and TP53 C176Y germline mutation in this AP case. The genomic evolution analysis showed different evolutionary branches in the metastatic tumors distinct from the primary lesion. Moreover, compared to the control group, the AP case showed high copy number variations (CNVs) and significantly high copy number instability (CNI). Conclusions: We speculated that the atypical histopathological features and malignant behaviors may be due to the co-mutations of somatic AKT1 E17K and germline TP53 C176Y, combined with the high CNVs and CNI.

\section{Introduction}

Pulmonary sclerosing pneumocytoma (PSP), formerly known as pulmonary sclerosing hemangioma, is a rare primary lung tumor initially described by Liebow et al, in 1956 as a tumor with prominent sclerosis and vascularization [1, 2]. It mainly affects adults $>50$ years of age, with a female to male ratio of $5: 1$ [3, 4]. Immunohistochemistry (IHC) markers clarified the epithelial origin of PSP and suggested that the condition originated from incompletely differentiated type II pneumocytes. Therefore, the term "pulmonary sclerosing hemangioma" was abandoned in the WHO 2016 classification for lung and pleura tumors, and PSP was adopted $[2,5,6]$. Although PSP has some clinical and imaging characteristics for differentiation from similar lesions, these are not specific for diagnosis [7, 8]. At present, the diagnosis of PSP still depends on surgical pathology. The primary pathological characteristics include the following: 1) The tumor is mainly composed of well-differentiated surface cuboidal epithelial cells and polygonal stromal cells. ${ }^{6}$ 2) Four common histological patterns can be seen, including papillary, solid, sclerotic, and hemorrhagic structures, or mainly composed of two patterns, which can co-exist in single tumor. 3) Immunohistochemically, the surface cells and stromal cells show differential expression. The stromal cells are diffusely positive for vimentin, TTF-1, and EMA, and always negative for CK, CK7, NapsinA, SP-B, and CEA, while the latter molecules are always positive in the surface cells. Occasionally, PSP may manifest as multiple lesions, recurrence, regional lymph node $(\mathrm{LN})$, or single organ metastasis, but does not seem to affect the prognosis. Therefore, PSP is considered as a benign or a low-grade malignant potential primary lung tumor [9-14].

The molecular alterations in PSP have been investigated in a few studies. A large-scale study using whole-exome sequencing (WES) identified a high frequency of $A K T 1$ point mutations $(31 / 68,46 \%)$ in PSP samples [15]. Recurrent internal tandem duplications in the $A K T 1$ gene in $22 / 44$ tumor samples (50\%) indicated that it was mutually exclusive of the other forms of $A K T 1$ mutations, including point mutations and short InDels (insertions and deletions). The AKT1 mutation rate, taken together all mutation forms, in almost all the PSP samples was $96 \%$ (81/84 cases) [16]. These results demonstrated that $A K T 1$ mutation is the hallmark of PSP, and internal tandem duplications and point mutations were the most common forms.

Nevertheless, the rapid recurrence of PSP, followed by multiple LNs and organ metastases, has never been reported. Also, the molecular mechanism of the malignant progression is yet lacking. Based on the current atypical PSP case (AP), we aimed to elucidate this mechanism of malignant transformation of PSP through clinicopathological, WES, and IHC data.

\section{Methods}

\section{Patients and samples}

Formalin-fixed and paraffin-embedded (FFPE) tissues from the AP case and 5 classic PSP cases were obtained from patients diagnosed with pathologically verified PSP from the Department of Pathology of Daping Hospital (Chongqing, China) from July 9, 2018 to July 22, 2019. AP was a 23-year-old male diagnosed as PSP. Samples from the primary and metastatic lesions were used for further analysis. The control group consisted of specimens from 5 female patients (mean age, 53 years; age range, 43-63 years). The clinical and pathological data of the patients were obtained from medical records. All patients provided informed written consent to allow publication of their clinical data. The present study was approved by The Clinical Ethics Committee of Daping Hospital, Army Medical University (Chongqing, China).

\section{IHC and double-labelling IHC}

FFPE tissues were sliced into $4-\mu \mathrm{m}$-thick sections. The tissues were subsequently deparaffinized and pretreated with $1 \mathrm{mmol} / \mathrm{L}$ EDTA at pH 9.0 in a high-pressure cooker for 3 min and then treated with $3 \% \mathrm{H}_{2} \mathrm{O}_{2}$ for 10 min. Subsequently, washing was performed for 3 min three times using $0.01 \mathrm{M}$ PBS at room temperature, and the slides were incubated with primary antibodies (Supplemental table 1 ) in the humidified chamber at $4^{\circ} \mathrm{C}$ overnight. Next day, the slides were washed in 0.01M PBS and then incubated with goat anti-mouse/rabbit IgG ready-to-use reagent (PV-6000, ZSGB-BIO, China) at room temperature for $30 \mathrm{~min}$ and stained using 3,3'-diaminobenzidine for $5 \mathrm{~min}$ at room temperature. Double-labelling IHC: after the staining of 3,3'-diaminobenzidine (PR or TTF-1), the slides were washed using $0.01 \mathrm{M}$ TBST for $3 \mathrm{~min}$ three times at room temperature, and incubated with antibody CK7 in the humidified chamber at $37^{\circ} \mathrm{C}$ for 1 hour. The slides were washed in $0.01 \mathrm{M} \mathrm{TBST}$ and then incubated with alkaline phosphatase horse anti-mouse IgG reagent (1:50, ZB-2310, ZSGB- 
$\mathrm{BIO}$, China) at room temperature for $30 \mathrm{~min}$ and stained using alkaline phosphatase substrate for 30 min at room temperature. The slides were reviewed using bright-field microscope (BX41, Olympus. 40x) and Cell Sens Standard software (version 1.16, Olympus) by pathologists.

\section{DNA extraction, library preparation and WES analysis}

WES and bioinformatic analysis of tumor specimens were conducted at a CAP-certified laboratory (GeneCast Biotechnology Co., Beijing). For each patient, archived FFPE blocks of tumor biopsies were used for DNA extraction. Genomic DNA was extracted from FFPE sections using the BLACK PREP FFPE DNA kit (AnalytikJena) according to the manufacturer's protocol. The quantity and quality of the extracted DNA were evaluated using a Qubit 3.0 fluorometer(Thermo Fisher Scientific)and Bioanalyzer 2100 (Agilent Technologies). DNA was fragmented using a Covaris M220 sonication system (Covaris) to obtain 200 bp fragments and purified using Agencourt AMPure XP beads (Beckman Coulter). Library preparations of the fragmented DNA were performed using the KAPA Hyper Prep Kit (KAPA Biosystems), following the manufacturer's protocol. Libraries with different indices were pooled for Hypercap Target Enrichment Kit(Roche), and SeqCap EZ MedExome kit(Roche) covering the exome genes. The captured library was further amplified using Illumina p5 (5' AAT GATACG GCG ACC ACC GA 3') and p7 (5' CAA GCA GAAGAC GGC ATA CGA GAT 3') primers in the KAPA Hifi-Hot Start Ready Mix (KAPA Biosystems), and purified with Agencourt AMPure XP beads. Sequencing libraries were quantified by Bioanalyzer 2100 (Agilent Technologies). The final libraries were sequenced on an Illumina Novaseq 6000 platform to a mean coverage depth of at least 100x, following the manufacturer's instructions.

\section{Statistics analyses}

The bioinformatic pipeline for WES analysis attached as the supplemental data. Copy number variations (CNVs) calling was determined using CONTRA paired method [17]. The genome DNA copy number instability (CNI) was calculated by the method described before [18] and the results were considered statistically significant at a $p$ value less than 0.05.LICHeE software was used for clonal evolution analysis based on the somatic mutations [19]. Venn diagram was drawn by the venn diagram package of $\mathrm{R}$ software [20].

\section{Results}

\section{Clinical history}

AP presented non-productive cough, chest distress, and fever for one month. He had no smoking and family disease history. Chest enhanced computed tomography (CT) scans confirmed a $6.5-\mathrm{cm}$ pulmonary nodule in the right middle lobe (RML), with enlarged mediastinal LN (Fig. 1A-B). Positron-emission tomography (PET) showed increased uptake in the nodule and confirmed multiple mediastinal LNs (Fig. 1C-D), with no other areas of uptake. The blood examination showed that carcinoembryonic antigen (CEA $8.4 \mathrm{ng} / \mathrm{mL}$, the normal range $0.0-5.0 \mathrm{ng} / \mathrm{mL}$ ) and CA153 $(274.55 \mathrm{U} / \mathrm{mL}$, the normal range 0.0 $31.3 \mathrm{U} / \mathrm{mL}$ ) were abnormal. The transthoracic radiology-guided biopsy considered epithelial tumor, but PSP was preferred. The patient underwent thoracoscopic RML lobectomy and systematic LNs dissection on July 27,2018 . Four months after the initial operation, the patient presented productive bloody cough. Chest enhanced CT scans showed an upper paratracheal LN measuring $3.2 \mathrm{~cm} \times 2.2 \mathrm{~cm}$. The second thoracoscopic mediastinal lymphadenectomy was performed on November 20, 2018. On February 2019, three months after the second operation, neck-enhanced magnetic resonance imaging (MRI) showed an enlarged right supraclavicular LN measuring $5.4 \mathrm{~cm} \times 3.8 \mathrm{~cm}$ (Fig. 1E). The abdominal enhanced MRI showed a $1.5 \mathrm{~cm} \times 1.2 \mathrm{~cm}$ nodule in the upper left lobe of the liver (Fig. 1F). The patient underwent right neck mass resection on February 27, 2019, and laparoscopic liver mass resection on March 27, 2019.All the postoperative pathological examinations confirmed the diagnosis of PSP.

\section{Histopathologic findings confirmed the diagnosis of PSP with multiple LNs and liver metastases}

A large solid mass was found in the RML. The single tumor was well-demarcated and circular, with grayish-white, slightly tough texture cut surface, measuring $6 \mathrm{~cm}$ in diameter (Fig. 2A). Microscopically, the boundary between the tumor and the surrounding lung tissue was relatively clear except that a few minor infiltrative nodules were observed outside the main body of the tumor (Fig. 2B). Solid cell area, papillary structure and sclerotic area composed of different proportion of surface epithelioid cells and polygonal stromal cells constitute the common mixed growth pattern of PSP (Fig. 2C). The papillary structures in sclerosing background (Fig. 2E), as well as the transition zone of solid and papillary pattern (Fig. 2D),were shown, respectively. Focally in solid cell area, polygonal tumor cells are diffusely arranged, the cell density is increased, and some pleomorphism and atypia appear, but the mitosis is difficult to find (Fig. 2F and G). Focal tumor necrosis was noticed (Fig. 2H). Tumor thrombus was found in some blood vessels in the peripheral region of the tumor (Fig. 2P, box). IHC revealed that both the stromal cells and cuboidal surface cells were diffuse positive for EMA and TTF-1 (Fig. 2J), whereas only the surface cells expressed CK (Fig. 2I), CEA, and NapsinA (Fig. 2K). Double-labelling IHC (Fig. 2L and M) showed CK7 and TTF-1 positive, PR negative in surface-lining cells; whereas CK7 negative, TTF-1 and PR positive in stromal cells. Most of the tumor cells were p53-positive (indicating TP53 mutation, Fig. 2N), whereas only a few cells expressed CD56, Syn, and ER. Immunostaining for S-100, actin, desmin, HMB45, p63 and TFE3 were negative. The Ki-67 labeling index (LI) was about $5 \%$ (Fig. 20). ERG staining outlined the blood vessels and set off the tumor thrombus in vessels (Fig. 2P). The IHC results of 5 classic PSP cases as control were consistent with those of AP except that p53 was negative and Ki-67 LI was 0-2\%.

The histopathological and IHC examination of the resected specimens confirmed multiple organ metastasis of hilar, mediastinum (Fig. 3A-E), cervical LNs, and liver (Fig. 3F-J). Strikingly, the normal structures of LNs and the liver were partially destroyed. The polygonal tumor cells showed diffuse or patchy infiltration, increased pleomorphism and atypia, with intranuclear inclusions and tumor giant cells. Interstitial vascular hyperplasia and focal necrosis were easily identified. The immuno-phenotypes of the tumor cells were similar to that of stromal cells of the primary lung tumor: diffuse expression of EMA, TTF-1 (Fig. 3C and H), PR (Fig. 3D and I) and p53 (Fig. 3J), but no expression of CK7 and NapsinA (Fig. 3C, red box). The Ki-67 LI of mediastinum LNs and liver metastasis was higher than that of the primary lesion (25\% vs $5 \%$ ) (Fig. 3E). 
Herein, we conducted a comprehensive examination of genetic alterations (somatic mutations) in FFPE samples from 6 cases of PSP using WES: 4 samples from the primary and three metastatic lesions of AP with matched adjacent normal tissue and 5 samples from the classic PSP cases in the control group. A high frequency of $A K T 1$ E17K mutations was detected in primary lung tumor, mediastinal LN, cervical LN, and liver metastatic lesions of AP (16.14\%, 16.83\%, $34.51 \%$, and $21.88 \%$, respectively). AKT1 gene mutations were detected in 3/5 classic PSP cases, including missense mutations (W80R + Q79K) in 2 cases and an internal tandem duplication (R76_C77insWPNTFIIR) in 1 case. Wild-type AKT1 genes were identified from the other two classic PSP cases (Fig. 4A, Table 1).

Table 1

Clinical data, AKT7and TP53 gene mutations in samples of AP and benign PSP cases.

\begin{tabular}{|c|c|c|c|c|c|c|c|c|c|}
\hline Group & $\begin{array}{l}\text { Tumor_sample } \\
\text { No. }\end{array}$ & Gender & Age & Chr & Type & $\begin{array}{l}\text { Freq } \\
(\%)\end{array}$ & Gene & Mutation_c & Mutation_p \\
\hline \multirow[t]{7}{*}{ Control } & 1920242_2 & Female & 63 & chr14 & SNV & 33.52 & AKT1 & c. $238 \mathrm{~T}>\mathrm{A}$ & p.W80R \\
\hline & & & & & & 33.24 & & c. $235 \mathrm{C}>\mathrm{A}$ & p.Q79K \\
\hline & $1922263-2$ & Female & 49 & chr14 & - & - & AKT1 & - & - \\
\hline & 1921057_3 & Female & 43 & chr14 & SNV & 9.85 & AKT1 & c. $238 \mathrm{~T}>\mathrm{A}$ & p.W80R \\
\hline & & & & & Complex & 11.00 & & c.234_235delinsAA & p.Q79K \\
\hline & $1818488-2$ & Female & 49 & chr14 & - & - & AKT1 & - & - \\
\hline & 1917145_2 & Female & 63 & chr14 & Insertion & 5.99 & AKT1 & c.207_230dupGCCCAACACCTTCATCATCCGCTG & p.R76_C77insWPN \\
\hline \multirow[t]{4}{*}{ AP } & AP1820610_3 & Male & 23 & chr14 & SNV & 16.14 & AKT1 & c. $49 \mathrm{G}>\mathrm{A}$ & p.E17K \\
\hline & AP1832384_3 & & & chr14 & SNV & 16.83 & AKT1 & c. $49 \mathrm{G}>\mathrm{A}$ & p.E17K \\
\hline & AP1905401_2 & & & chr14 & SNV & 34.51 & AKT1 & c. $49 \mathrm{G}>\mathrm{A}$ & p.E17K \\
\hline & AP1909075_2 & & & chr14 & SNV & 21.88 & AKT1 & c. $49 \mathrm{G}>\mathrm{A}$ & p.E17K \\
\hline \multirow[t]{5}{*}{ AP } & AP-Normal & Male & 23 & chr17 & SNV & 33.02 & TP53 & c. $527 \mathrm{G}>\mathrm{A}$ & p.C176Y \\
\hline & AP1820610_3 & & & chr17 & SNV & 42.41 & TP53 & c. $527 \mathrm{G}>\mathrm{A}$ & p.C176Y \\
\hline & AP1832384_3 & & & chr17 & SNV & 68.63 & TP53 & c. $527 \mathrm{G}>\mathrm{A}$ & p.C176Y \\
\hline & AP1905401_2 & & & chr17 & SNV & 72.84 & TP53 & c. $527 \mathrm{G}>\mathrm{A}$ & p.C176Y \\
\hline & AP1909075_2 & & & chr17 & SNV & 50.52 & TP53 & c. $527 \mathrm{G}>\mathrm{A}$ & p.C176Y \\
\hline
\end{tabular}

PSP, pulmonary sclerosing pneumocytoma; AP, atypical PSP; Chr, chromosome; Freq, frequency; SNV, single nucleotide variation.

TP53 $\mathrm{C} 176 \mathrm{Y}$ mutations were identified in the primary lung lesion, mediastinal LN, cervical LN, and liver metastatic samples of AP, with the frequency $42.41 \%$, $68.63 \%, 72.84 \%$, and $50.52 \%$ respectively. Interestingly, TP53 C176Y mutations were also detected in the paired normal tissue adjacent to the primary lung tumor (frequency $33.02 \%$ ), suggesting that TP53 C176Y was a germline mutation in this metastatic PSP patient (Fig. 4A, Table 1). This phenotype was further confirmed by the identification of the same mutation in the peripheral blood mononuclear cell (PBMC) sample (Supplemental Fig. 1).

\section{Genomic evolution analysis and copy number instability of AP}

In order to demonstrate the malignant progression of this case, we analyzed the mutation evolution differences between the primary foci and three metastatic lesions based on the data of WES. These foci and lesions carried three "trunk mutations": AKT1 E17K, NCOR1 R190_E191delins*, and COL2A1 P1183H. Along with disease progression, the three metastatic lesions evolved an $\angle R P 1 B$ W1304C mutation, which was the main difference from the primary tumor. Furthermore, the mediastinal LN, cervical LN, and liver metastases had been identified with DROSHA P19S (M1), SMO L23delL (M2), and RECQL V41Sfs*14 (M3) mutations respectively, which constituted independent branches of the evolutionary tree (Fig. 4B-D).

Based on the WES data, genomic DNA copy number variation (CNV) analysis was performed on four lesions of AP and 5 samples of the control group. A higher number of CNVs were detected in the AP samples than the control group. Genes with high abundance of CNVs in the samples of AP included SP8, MIB2, LOC101927859, FAM230A, and ACAP3. Also, differences were noted among the four lesions of AP: the mediastinal LN (AP1832384), cervical LN (AP1905401), and hepatic metastatic lesion (AP1909075) harbored a higher number of CNVs than the primary tumor (AP1820610) (Fig. 4E). In addition, a significantly higher copy number instability $(\mathrm{CNI})$ was observed in the AP samples than the control group $(P=0.02)(\mathrm{Fig}$. 4F). The tumor mutational burden was 2.3 Muts/Mb (TMB-L) calculated based on the WES data.

\section{Clinical outcome after the fourth operation}

Due to the high level of serum prolactin with positive estrogen receptor (ER) and progesterone receptor (PR) in operative FFPE tissues as assessed by IHC, the patient received antiestrogen therapy with medroxyprogesterone after the operation. In May 2019, chest and abdominal enhanced CT scans showed dramatic progression: multiple nodules in the right lung and multiple enlarged LNs in neck, jaw and right supraclavicular fossa. Since the antiestrogen therapy was ineffective, the patient stopped taking medroxyprogesterone orally. 
In September 2019, the patient felt that the neck mass was significantly larger than before. Chest and abdominal enhanced CT scans (Fig. 5A-D) showed an enlarged mass in both lung hilum $(5.8 \mathrm{~cm} \times 5.4 \mathrm{~cm})$ and right supraclavicular fossa $(5.7 \mathrm{~cm}$ in maximum dimension), multiple nodules in the liver and left kidney, and thoracic 8th and11th vertebral osteolytic bone destruction. The patient was administered pembrolizumab treatment at $2 \mathrm{mg} / \mathrm{kg}$ intravenously every 3 weeks, combined with apatinib at $250 \mathrm{mg} /$ day orally since October 2, 2019. After 2 cycles of combination therapy, the patient reported feeling well, and the neck mass was significantly reduced.

On November 11, 2019, chest enhanced CT showed a significant shrinkage in the volume of multiple lesions including right supraclavicular fossa, hilum, mediastinum, and liver (Fig. 5E-H). The patient subsequently received another three cycles of the same treatment, achieving a partial response.

\section{Discussion}

Due to the lack of specificity in clinical and imaging features, the diagnosis of PSP mainly depends on postoperative pathological examination. The diagnosis of typical PSP, characterized by two different cell components, four typical histological types, and immunophenotypes with specific features, was not difficult for pathologists [6]. However, some atypical cases showed either different clinical behaviors from typical PSP, such as recurrence or metastasis, or confused histological characteristics, such as limited typical patterns, cytological atypia, and focal necrosis. These atypical cases could be misdiagnosed as papillary or solid subtype of lung adenocarcinoma and neuroendocrine tumor, especially in the case of needle biopsy or intraoperative frozen diagnosis [21]. In this case, the delayed diagnosis at biopsy was mainly attributed to the atypical phenomenon, including multiple LN metastases, papillary and solid growth pattern, cellular atypia, tumoral necroticfoci, and higher Ki-67 LI than that of ordinary PSP cases. Therefore, for accurate conclusions, we should comprehensively analyze the results of imaging, gross specimen performance, histopathology, IHC, and molecular detection, if necessary.

There are several points worth emphasizing on the pathological diagnosis. Firstly, PSP usually presents as an isolated, solid, and well-defined mass, which is different from the general changes of invasive adenocarcinoma. Secondly, we should deduce the basic structure for the diagnosis of PSP by observing the sections carefully and comprehensively: the papillary growth pattern and its diffuse distribution of interstitial cell components, as well as other histological patterns, such as solid cell area, intra-alveolar hemorrhage, and sclerotic changes. Thirdly, although there is no specific single antibody for diagnosis, establishing the diagnosis by the appropriate combination of antibodies and observing the obvious difference of immunophenotype between epithelioid cells and stromal cells is imperative. Finally, for the atypical cases, the molecular pathologic finding is helpful for differential diagnosis. As in this case, we identified the AKT1 E17K point mutation through WES analysis, which is considered to be a relatively specific molecular feature of PSP [15, 16], but no other common driving gene mutations related to lung cancer were found, which plays an important role in strengthening our confidence in the diagnosis of this atypical disease.

PSPs were mostly diagnosed in female patients (83.34\%), aged 38-61 years [22]. The malignant progression and metastasis of PSP were extremely rare. The reported PSP patients with $\operatorname{LN}[9,13,14,23]$ or organ metastases $[11,24]$ were mostly females, and only one male patient suffered from mediastinal LN metastasis [25]. Herein, we reported an extremely atypical case: a young male patient suffering from an aggressive PSP with multiple LN and organ metastases during 7 months after the resection of the primary lung lesion.

AKT1 E17K mutation, identified from all primary and metastatic lesions in this study, were localized to the pleckstrinhomology domain (PH domain), which is crucial for membrane localization and downstream activation of $A K T 1$ [26]and is known to promote growth factor-independent cell proliferation [27, 28].

However, 3 PSP cases in this study with mutations on the PH domain of the AKT1 gene (Q79K, W80R, and R76_C77insWPNTFIIR) did not show any malignant progression. Moreover, though more than 40 PSP cases have been reported with AKT1 E17K mutation [15, 16], malignant progression has been rarely reported. Taken together, these results indicated that single E17K mutation on the $\mathrm{PH}$ domain of the $A K T 1$ gene might not be sufficient to initiate the malignant transformation of the tumor. A 17-year-old girl suffering from multiple nodules in the right lung lobe diagnosed as PSP with both AKT1 E17K and BRAF V600E mutations [29]. The other PSP case with diffusely scattered nodules in the right lung, harbored AKT1 E17K and other 14 somatic gene mutations [30]. These indicated that the combination of $A K T 1$ mutations with other oncogenes might accelerate the malignant progression of benign PSPs. For the first time, we reported a TP53 C176Y germline mutation, a likely pathogenic mutation according to the ClinVar and 1000Genomes database, in this extremely aggressive PSP case. Which consistent with the positive expression of the P53 mutant protein in the IHC assay. Therefore, rapid malignant progression leading to multiple metastases of this PSP case might be partially attributed to the combination of somatic AKT1 E17K mutation and germline TP53 C176Y mutation. Germline mutations of TP53 gene have been identified in $80 \%$ of patients with Li-Fraumeni syndrome (LFS), a cancer predisposition syndrome associated with high risks for a diverse spectrum of childhood-and adult-onset malignancies [31]. However, neither first- nor second-degree relatives had been diagnosed with any cancer or sarcoma, this patient does not meet the classic LFS diagnosis criteria [32].

Evolutionary analysis revealed distinct molecular biological variations at different malignant progression stages of this case. Among these molecular variations, NCOR1 R190_E191delins* and RECQL V41sfs*14 might be the two additional important mutations that are responsible for malignancy. Nuclear receptor co-repressor 1 (NCOR1) is a transcriptional regulator bridging repressive chromatin-modifying enzymes with transcription factors [33]. As a putative tumor suppressor and independent prognostic factor, the NCOR1 mutations resulted in embryonic lethality [34] and cell type-and tissue-specific functions in multiple cells and organs [35-38]. The mutation of NCOR1 R190_E191delins*leads to the premature transcriptional termination, followed by loss of function, which might generate excessive transcription. RECQL is also known as RECQL1 or RECQ1, one of the members of human RECQ helicase family [39]. The RECQL helicases that unwind dsDNA are involved in several important cellular functions, including DNA repair, replication, recombination, and transcription [40]. The phenotypes observed in RECQL loss-function mouse and human cells suggested its role in the maintenance of genome integrity, chromosomal stability and in the non-homologous end-joining DNA repair [41-43]. Mutations of some human RECQ helicases cause different heritable cancer susceptibility syndromes, such as Werner syndrome and Bloom syndrome [44]. These results indicated a tumor-suppressor role for RECQL. We identified RECQL gene V41sfs*14 mutation in the liver metastasis lesion. This loss-of-function mutation might result in the premature transcription termination of RECQL and high CNI of the DNA genome. 
CNVs constitute a major source of variations among humans, underlying human evolution, many mental illnesses, developmental disorders, and cancers [45]. CNVs for NSCLC distinguished lung cancer from normal lung tissues, can be a prognostic indicator [46]. A number of low-to-common frequency CNVs might influence the risk of epithelial ovarian cancer and tumor-gene expression [47]. DNA copy number losses in basal-like breast cancers were associated with significantly increased genomic instability and poor patient survival [48]. Herein, a higher number of CNVs were identified in the AP samples as compared to the control group and in metastatic lesions than the primary tumor of AP. Furthermore, the CNI was significantly higher in the AP samples than the control group. These factors could be ascribed to the rapid malignant progression of this PSP case. Although statistical bias may exist due to the limited number of cases, such significant differences in CNI values between different groups of the same disease are rather uncommon.

\section{Conclusion}

We reported an extremely rare case of PSP, which showed obvious atypical features in histopathology and malignant biological behaviors, such as rapid recurrence and multiple metastases. Based on the results of WES, we speculated that the somatic AKT1 E17K and germline TP53 C176Y mutations, combined with high CNVs and CNI might account for this malignant progression.

\section{Abbreviations}

\section{AP}

Atypical PSP

CNI

Copy number instability

CNVs

Copy number variations

CT

Computed tomography

FFPE

Formalin-fixed and paraffin-embedded

IHC

Immunohistochemistry

\section{LNs}

Lymph nodes

MRI

Magnetic resonance imaging

PET

Positron-emission tomography

PSP

Pulmonary sclerosing pneumocytoma

RML

Right middle lobe

WES

Whole-exome sequencing

\section{Declarations}

\section{Ethics approval and consent to participate}

The study was approved by The Clinical Ethics Committee of Daping Hospital, Army Medical University (Chongqing, China). All patients signed the consent to allow publication of their clinical data.

\section{Consent for publication}

All authors read and approved the final manuscript.

\section{Funding}

This work was supported by grants from the Daping Hospital, Army Medical University.

\section{Competing interests}

The authors declare that they have no conflict of interest.

\section{Contributions:}

QSW and HLX designed the study. XY and MRJ investigated and provided the clinical data. QSW, LZ and YY performed the molecular experiments. LHZ, QM and YC diagnosed the cases. CYM and PF performed the pathologic slides. All authors analyzed the data. QSW and HLX wrote the manuscript. MXL, YH and 
HLX revised and edited the manuscript. All authors read and approved the final manuscript.

\section{References}

1. Liebow AA, Hubbell DS. Sclerosing haemangioma (histiocytoma, xanthoma) of the lung. Cancer. 1956; 9:53-75.

2. Alshelli I, Seeley EJ. Pulmonary Sclerosing Pneumocytoma: Did Lightening Just Strike Twice? Semin Thorac Cardiovasc Surg. 2017; 29(4):561-562.

3. Devouassoux-Shisheboran M, Hayashi T, Linnoila RI, et al. A clinicopathologic study of 100 cases of pulmonary sclerosing hemangioma with immunohistochemical studies: TTF-1 is expressed in both round and surface cells, suggesting an origin from primitive respiratory epithelium. Am $J$ Surg Pathol. 2000; 24: 906-916.

4. Illei PB, Rosai J, Klimstra DS. Expression of thyroid transcription factor-1 and other markers in sclerosing hemangioma of the lung. Arch Pathol Lab Med. $2001 ; 125: 1335-1339$

5. Gaucher L, Patra P, Despins P, et al. A rare tumor: Benign sclerosing pneumocytoma with an intrascissural development. Poumon Coeur.1983; 39(6):321326.

6. Travis WD, Brambilla E, Nicholson AG, et al. The 2015 World Health Organization Classification of Lung Tumors: Impact of Genetic, Clinical and Radiologic Advances Since the 2004 Classification. J Thorac Oncol. 2015;10:1243-1260.

7. Shin SY, Kim MY, Oh SY, et al. Pulmonary sclerosing pneumocytoma of the lung: CT characteristics in a large series of a tertiary referral center.Medicine (Baltimore). 2015; 94(4):e498.

8. Zhu, J. Analysis of the clinical differentiation of pulmonary sclerosing pneumocytoma and lung cancer. J Thorac Dis. 2017; 9(9): 2974-2981.

9. Pokharel S, Dhillon SS, Ylagan L, et al. Sclerosing Pneumocytoma with Lymph Node Metastasis. J Thorac Oncol. 2016; 11(10): 1802-1804.

10. Arumugam VG, Joseph LD, Thangavel P, et al. Sclerosing pneumocytoma of the lung: a case report. J Clin Diagn Res.2017; 11 (2):ED12-14.

11. Kim MK, Jang SJ, Kim YH, et al. Bone metastasis in pulmonary sclerosing hemangioma. Kor J Intern Med. 2015; 30(6):928-930.

12. Xu HM, Zhang G. A rare case of pulmonary sclerosing hemagioma with lymph node metastasis and review of the literature. Int J Clin Exp Pathol.2015; 8(7):8619-8623.

13. Soo IX, Sittampalam K, Lim CH. Pulmonary sclerosing pneumocytoma with mediastinal lymph node metastasis. Asian Cardiovasc Thorac Ann. 2017; 25(7-8):547-549.

14. Wang X, Zhang L, Wang Y, et al. Sclerosing pneumocytoma with metastasis to the mediastinal and regional lymph nodes. Indian J Pathol Microbiol. 2018; 60(3):407-409.

15. Jung SH, Kim MS, Lee SH, et al. Whole-exome sequencing identifies recurrent AKT1 mutations in sclerosing hemangioma of lung. Proc Natl Acad Sci USA. $2016 ; 113: 10672-10677$

16. Yeh YC, Ho HL, Wu YC, et al. AKT1 internal tandem duplications and point mutations are the genetic hallmarks of sclerosing pneumocytoma. Mod Pathol. 2020;33(3):391-403.

17. Li J, Lupat R, Amarasinghe KC, et al. CONTRA: copy number analysis for targeted resequencing. Bioinformatics. 2012; 28(10):1307-1313.

18. Weiss GJ, Beck J, Braun DP, et al. Tumor Cell-Free DNA Copy Number Instability Predicts Therapeutic Response to Immunotherapy. Clin Cancer Res. 2017; 23(17):5074-5081.

19. Popic V, Salari R, Hajirasouliha I, et al. Fast and scalable inference of multi-sample cancer lineages. Genome Biology.2015;16:91.

20. Chen H, Boutros PC. VennDiagram: a package for the generation of highly-customizable Venn and Euler diagrams in R. BMC Bioinformatics. $2011 ; 12: 35$.

21. Yang $\mathrm{CH}$, Lee LY. Pulmonary sclerosing pneumocytoma remains a diagnostic challenge using frozen sections: a clinicopathological analysis of 59 cases. Histopathology. 2018; 72(3):500-508.

22. Lovrenski A, Vasilijević M, Panjković M, et al. Sclerosing Pneumocytoma: A Ten-Year Experience at a Western Balkan University Hospital. Medicina (Kaunas). 2019; 55(2):pii: E27.

23. Adachi Y, Tsuta K, Hirano R, et al. Pulmonary sclerosing hemangioma with lymph node metastasis: A case report and literature review. Oncol Lett. 2014; 7(4):997-1000.

24. Bae YS, Ro JY, Shim HS, et al. Pulmonary sclerosing haemangioma with metastatic spread to stomach. Histopathology. 2012; 60(7):1162-1164.

25. Katakura H, Sato M, Tanaka F, et al. Pulmonary sclerosing hemangioma with metastasis to the mediastinal lymph node. Ann Thorac Surg. 2005; 80(6):2351-2353.

26. Vivanco I, Sawyers CL. The phosphatidylinositol 3-Kinase AKT pathway in human cancer. Nat Rev Cancer. 2002; 2(7):489-501.

27. Parikh C, Janakiraman V, Wu WI, et al. Disruption of PH-kinase domain interactions leads to oncogenic activation of AKT in human cancers. Proc Natl Acad Sci USA. 2012; 109(47):19368-19373.

28. Carpten JD, Faber AL, Horn C, et al. A transforming mutation in the pleckstrin homology domain of AKT1 in cancer. Nature. 2007;448:439-444.

29. Jiang G, Zhang M, Tan Q, et al. Identification of the BRAF V600E mutation in a patient with sclerosing pneumocytoma: A case report. Lung Cancer. 2019;137:52-55.

30. Fan X, Lin L, Wang J, et al. Genome profile in a extremely rare case of pulmonary sclerosing pneumocytoma presenting with diffusely-scattered nodules in the right Cancer Biol Ther. 2018; 19(1):13-19.

31. Villani A, Shore A, Wasserman J, et al. Biochemical and imaging surveillance in germline TP53 mutation carriers with Li-Fraumeni syndrome: 11 year follow-up of a prospective observational study. Lancet Oncol. 2016; 17: 1295-1305.

Page $7 / 12$ 
32. Schneider K, Zelley K, Nichols K, et al. Li-Fraumeni Syndrome. In: Adam MP, Ardinger HH, Pagon RA, et al. eds. GeneReviews $\AA$ [Internet]. Seattle (WA): University of Washington, Seattle; 1999 [Updated 2019]. p.1993-2020.

33. Müller L, Hainberger D, Stolz V, et al. The corepressor NCOR1 regulates the survival of single-positive thymocytes. Sci Rep. 2017; 7(1):15928.

34. Jepsen K, Hermanson O, Onami TM, et al. Combinatorial roles of the nuclear receptor corepressor in transcription and development. 2000; 102(6):753-763.

35. Yamamoto H, Williams EG, Mouchiroud L, et al. NCoR1 is a conserved physiological modulator of muscle mass and oxidative function. Cell. 2011; 147:827-839.

36. Li P, Fan W, Xu J, et al. Adipocyte NCoR knockout decreases PPARgamma phosphorylation and enhances PPARgamma activity and insulin sensitivity. Cell. 2011; 147(4):815-826.

37. Li P, Spann NJ, Kaikkonen MU, et al. NCoR repression of LXRs restricts macrophage biosynthesis of insulin-sensitizing omega 3 fatty acids. Cell. 2013; 155(1):200-214.

38. Astapova I, Lee LJ, Morales C, et al. The nuclear corepressor, NCoR, regulates thyroid hormone action in vivo. Proc Natl Acad Sci USA. 2008; 105(49):19544-19549.

39. Seki M, Miyazawa H, Tada S, et al. Molecular cloning of cDNA encoding human DNA helicase Q1 which has homology to Escherichia coli Rec Q helicase and localization of the gene at chromosome 12p12. Nucleic Acids Res.1994; 22(22):4566-4573.

40. Sharma S, Doherty KM, Brosh RM. Mechanisms of RecQ helicases in pathways of DNA metabolism and maintenance of genomic stability. Biochem J.2006; 398(3):319-337.

41. Cybulski C, Carrot-Zhang J, Kluźniak W, et al. Germline RECQL mutations are associated with breast cancer susceptibility. Nature Genetics.2015; 47(6):643-646.

42. Sharma S, Stumpo DJ, Balajee AS, et al. RECQL, a member of the RecQ family of DNA helicases, suppresses chromosomal instability. Mol Cell Biol.2007; 27(5):1784-1794.

43. Parvathaneni S, Stortchevoi A, Sommers JA, et al. Human RECQ1 interacts with Ku70/80 and modulates DNA end-joining of double-strand breaks. PLoS ONE. 2013; 8(5):e62481.

44. Monnat RJ. Human RECQ helicases: roles in DNA metabolism, mutagenesis and cancer biology. Semin Cancer Biol. 2010; 20(5):329-339.

45. Hastings PJ, Lupski JR, Rosenberg SM, et al. Mechanisms of change in gene copy number. Nat Rev Genet. 2009; 10(8):551-564.

46. Qiu ZW, Bi JH, Gazdar AF, et al. Genome-wide copy number variation pattern analysis and a classification signature for non-small cell lung cancer. Genes Chromosomes Cancer. 2017; 56(7):559-569.

47. Reid BM, Permuth JB, Chen YA, et al. Genome-wide Analysis of Common Copy Number Variation and Epithelial Ovarian Cancer Risk. Cancer Epidemiol Biomarkers Prev. 2019;28(7):1117-1126.

48. Weigman VJ, Chao HH, Shabalin AA, et al. Basal-like Breast cancer DNA copy number losses identify genes involved in genomic instability, response to therapy, and patient survival. Breast Cancer Res Treat. 2012; 133(3):865-880.

\section{Figures}



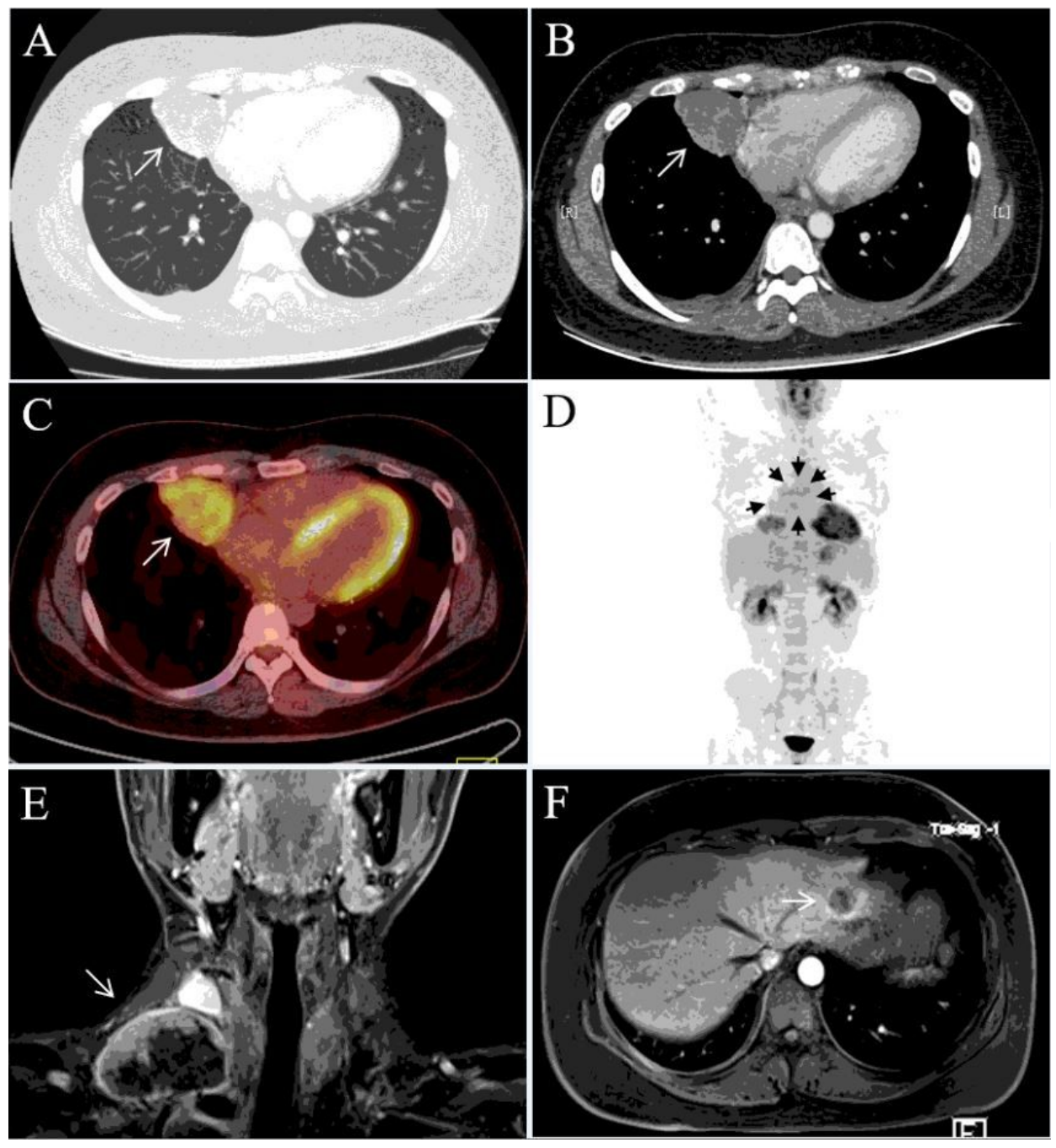

Figure 1

CT, PET and MRI scans showed a primary lesion in the right lung and metastatic tumors in mediastinum, right neck, and right liver. (A) The lung window of CT examination showed a round-like mass (arrow) in the medial segment of the right middle lobe with a smooth margin. (B) The lesions (arrow) in the enhanced arterial phase were slightly enhanced, and the tortuous small vessel shadow was seen inside. (C-D) PET examination showed that the radioactivity uptake of the right pulmonary pericardial lesions was increased (C, arrow), and the suspected annular radioactivity uptake in the mediastinum (D, arrows) was increased. No obvious abnormal radioactivity uptake was seen in other organs. (E) The neck MRI showed metastatic tumors (arrow) in the right neck after 5 months of the operation (thoracoscopic assisted resection of the right middle lung and systemic lymphadenectomy). The lesion was uneven enhancement and had abundant necrotic areas. (F) The abdomen MRI showed a lesion in the left lateral lobe of the liver (arrow). CT, chest enhanced computed tomography; PET, positron-emission tomography; MRI, magnetic resonance imaging. 

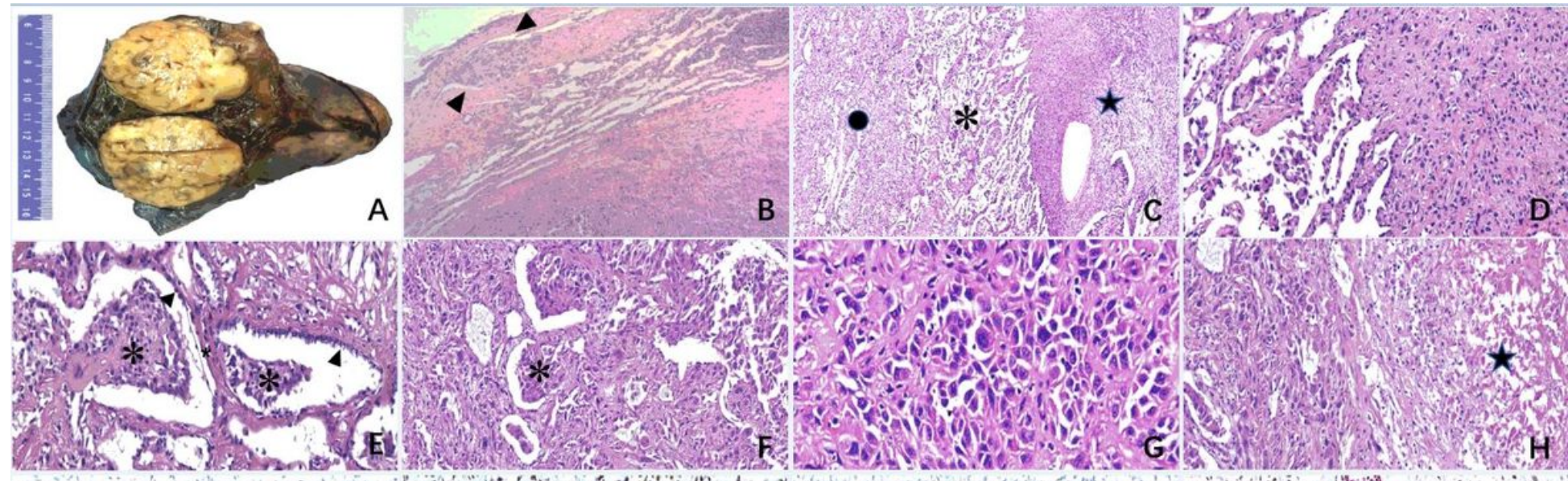

c

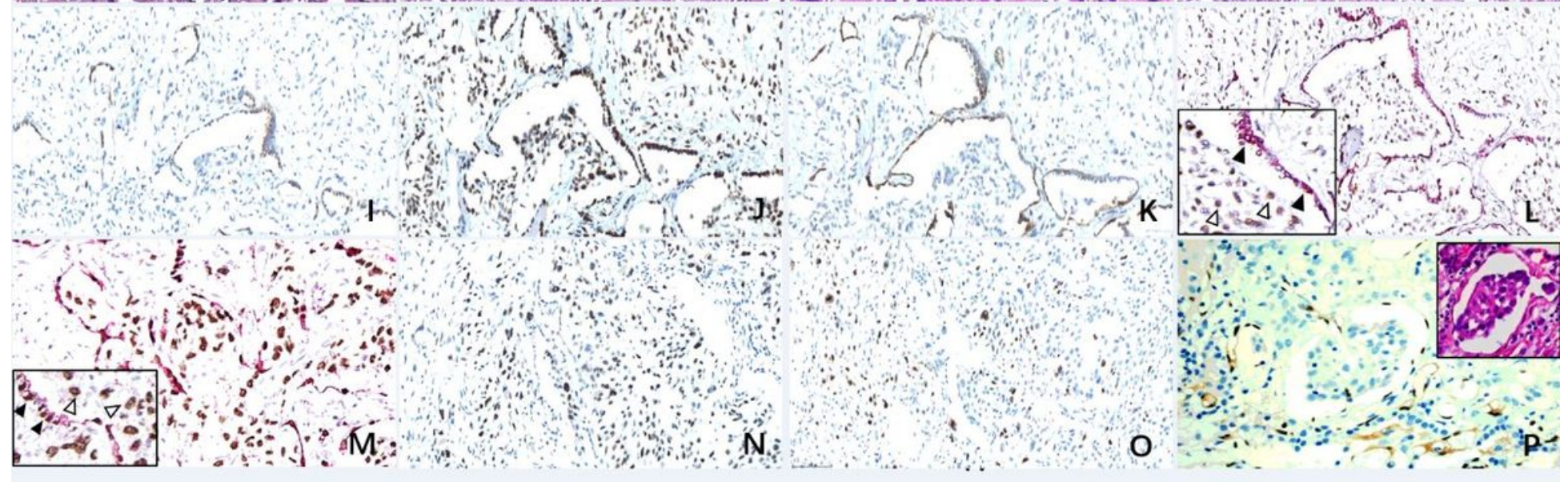

Figure 2

Histopathology of the primary pulmonary lesion in this unusual PSP case. (A) The gross examination showed a large solid mass in the middle right lobe with relatively clear boundary and grayish-white, slightly tough texture cut surface. (B) The boundary between tumor and normal lung tissue was relatively clear, with the invasion of minor tumor nodules (arrow). (C) Microscopically, there were solid cell area (star), papillary structure (asterisk) and sclerotic area (spot) in turn beneath normal lung tissue. (D) The migration of solid cell zone and papillary structures. (E) Papillary structures of different size (asterisk) covered with surface epithelial cells (arrow) appear in sclerotic background. (F) In the solid cell area, polygonal tumor cells were mainly diffusely arranged or forming papillary structures (asterisk). (G) The tumor cells with certain atypia and giant tumor cells could be seen, but mitosis was hardly to be found. (H) Focal tumoral necrosis could be seen (star). CK (I), TTF-1 (J) and NapsinA (K) expressed in the same region as illustrated in Fig 2(E). (L) Double-labelling IHC revealed that the surface epithelial cells (black arrow) are positive for CK7 and negative for PR, while the stromal-like cells (white arrow) in papillas are on the contrary. (M) Double labelling IHC showed CK7 and TTF-1 co-expressing in surface-lining cells (black arrow), while the stromal-like cells in papillas (white arrow) were only TTF-1 positive. P53 (N) and Ki-67 (O) expressed in the solid cell region and papillary structures as illustrated in Fig 2(F). (P) ERG staining outlined the shape of the blood vessels, showing tumor thrombus in them. PSP, pulmonary sclerosing pneumocytoma. IHC, immunohistochemistry.
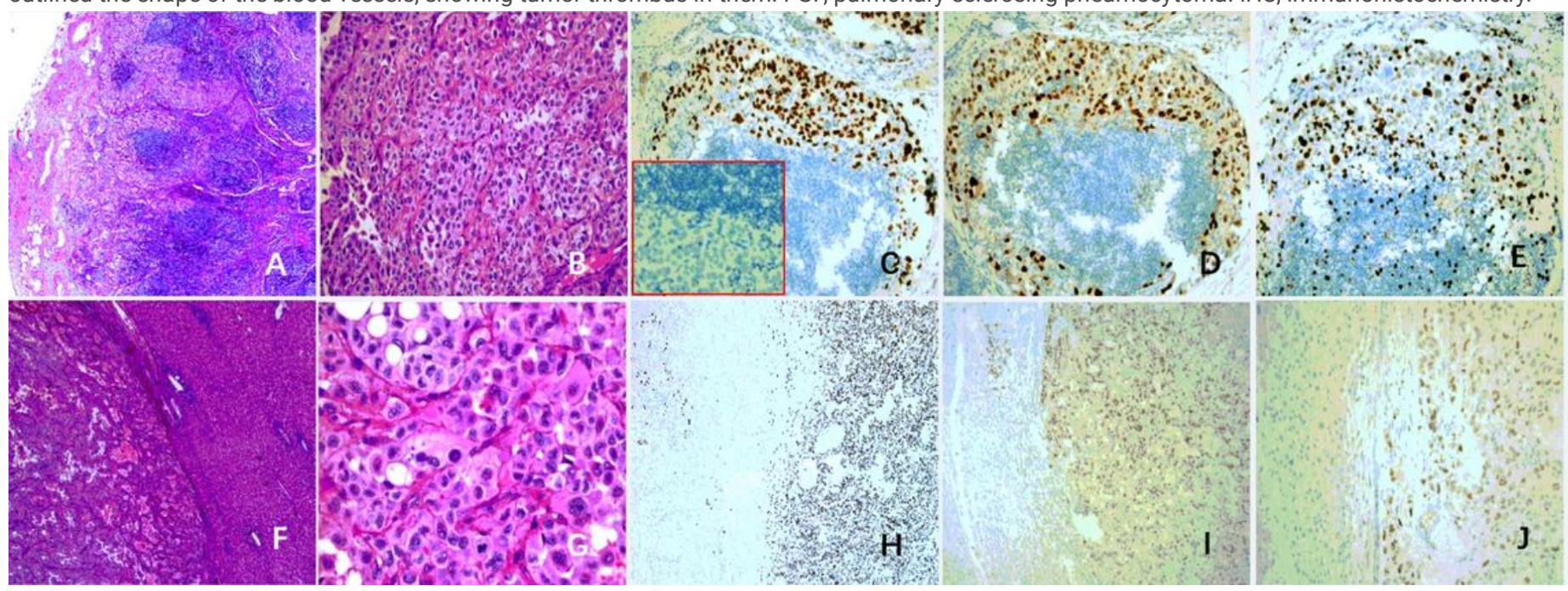

Figure 3 
Histopathological changes in metastatic lesions of AP. Tumors cells of mediastinal LNs (A, B) are positive for TTF-1 (C) and PR (D), but negative for NapsinA (C, red box). The $\mathrm{Ki}-67 \mathrm{LI}$ is about $25 \%(\mathrm{E})$. The tumor cells of liver metastasis $(\mathrm{F}, \mathrm{G})$ are similar to the polygonal stromal cells in the primary pulmonary lesion, with obvious pleomorphism and atypia, and the mitosis is not difficult to find (G). The tumor cells are positive for TTF-1(H), PR(I) and P53(J). AP, atypical PSP; LNs, lymph nodes; LI, labeling index.

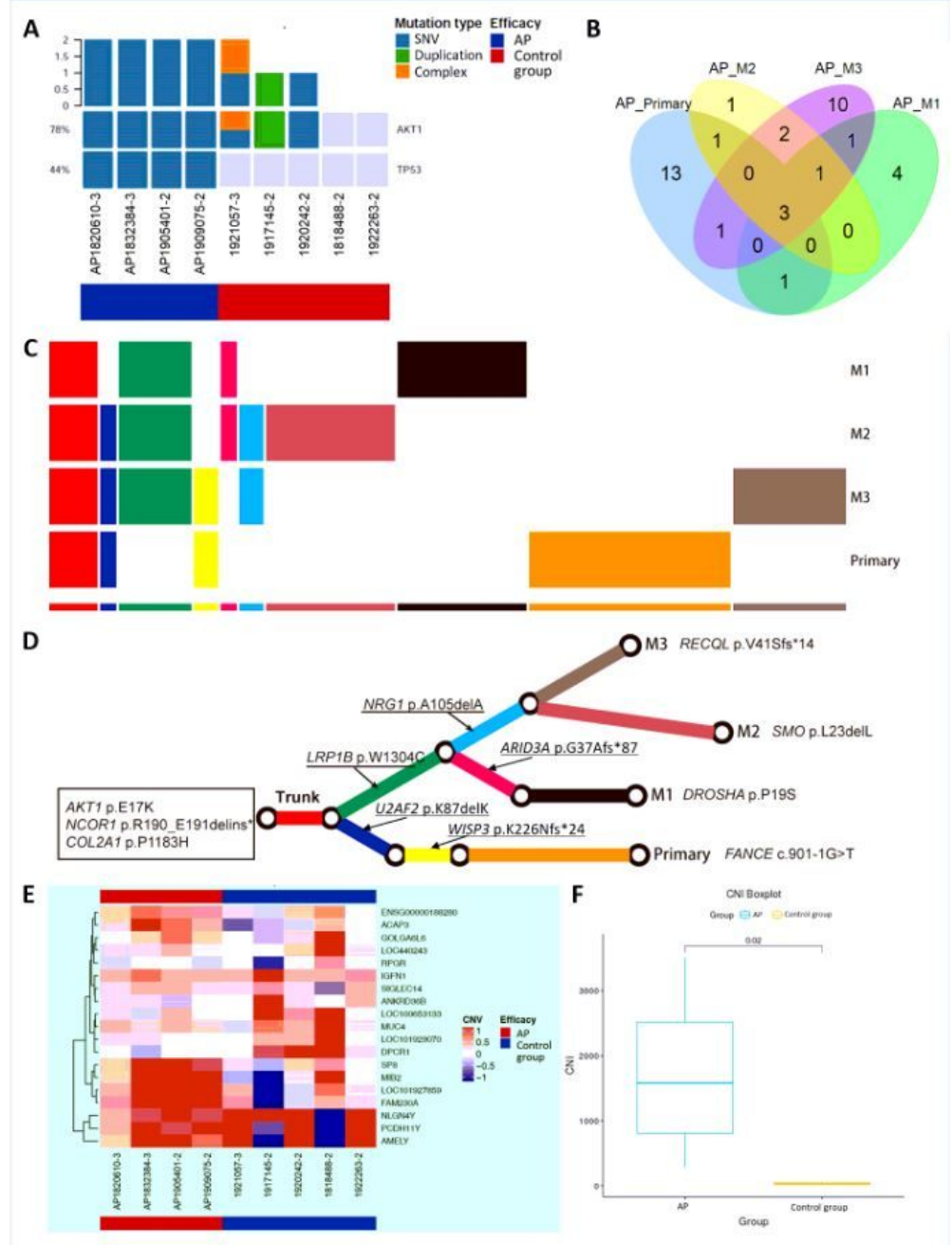

Figure 4

Molecular characteristics of this AP case based on the WES. (A) All the primary and metastatic tumors of AP harbored consistent AKT1 and TP53 singlenucleotide variations (SNVs). Only 3/5 classic PSP cases harbored AKT1 mutations. (B) Venn diagram and (C) heatmap showed the distribution of the mutations in the four lesions. (D) Clonal evolutionary analysis indicated that there were common and distinct evolutions in primary and three metastatic lesions. (E) CNV heatmap showed higher CNVs in four lesions of AP than in 5cases of the control group. (F) Samples of AP exhibited significantly higher CNI than that ofthe control group, $\mathrm{P}=0.02$. PSP, pulmonary sclerosing pneumocytoma; AP, atypical PSP; Primary, primary lung tumor; M1, mediastinal lymph node; M2, cervical lymph node; M3, liver metastasis; CNVs, copy number variations; CNI, copy number instability. 


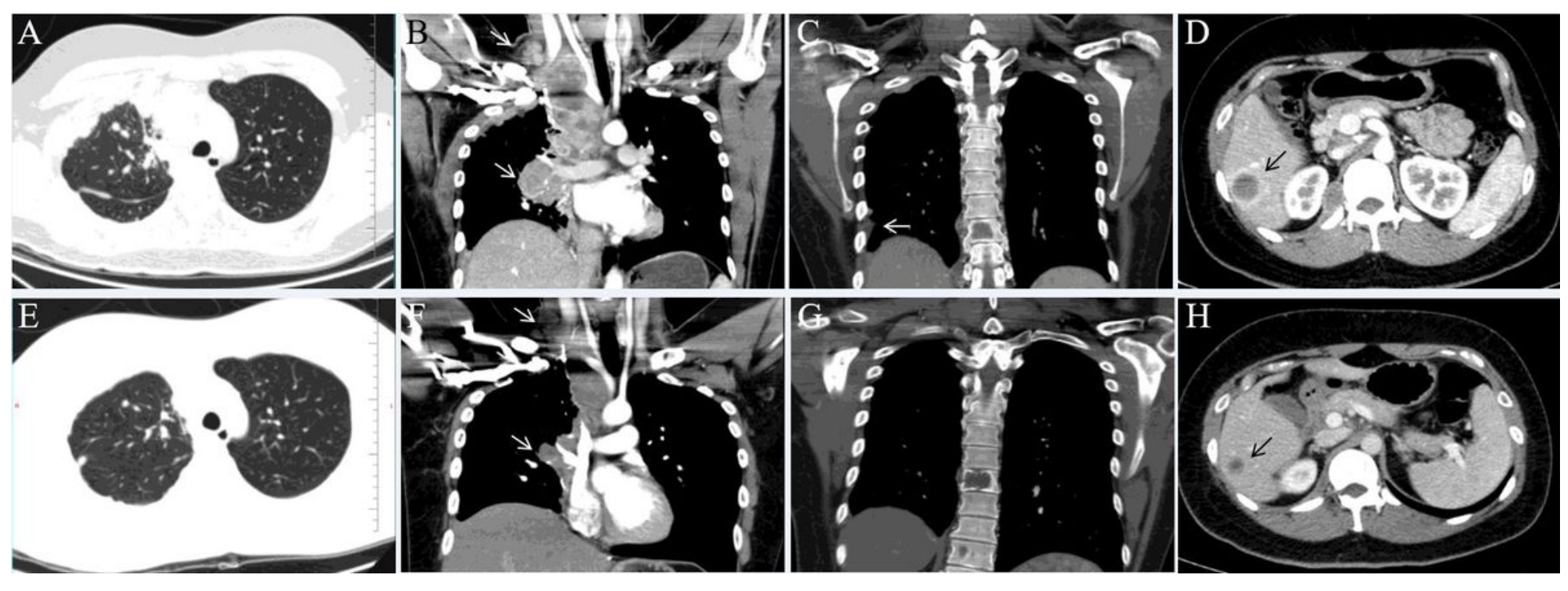

Figure 5

CT scans showed obvious shrinkage in both primary and metastatic lesions after four cycles of combined treatment. (A-D) In September 2019, CT scan showed the multiple metastatic lesions in the right lung hilum (A), right neck and mediastinum LNs (B). The right jugular vein and precava were invaded, the right pleura showed metastasis (C), and the liver showed new metastasis (D). (E-H) After the combined treatment (200 mg D1 of pembrolizumab and 250 $\mathrm{mg} /$ day of apatinib, four cycles), the chest and abdomen CT displayed a shrunk nodule in the upper lobe of the right lung (E). Lesions in the right neckand mediastinum LNs were shrunk (F). The lesion of the vertebral body was increased slightly $(\mathrm{G})$. The liver metastasis was shrunk (H). CT, chest enhanced computed tomography; LN, lymph node.

\section{Supplementary Files}

This is a list of supplementary files associated with this preprint. Click to download.

- Supplementalfigure1.jpg

- Supplementaldata.docx 